\title{
Comparative Analysis of Air-gap PD Characteristics: Vegetable Oil/pressboard and Mineral Oil/pressboard
}

\author{
Lu Cui ${ }^{1,2}$, Weigen Chen ${ }^{1}$, A. S. Vaughan ${ }^{2}$, Bo Xie ${ }^{1}$, Zhenze Long ${ }^{3}$ \\ ${ }^{1}$ State Key Laboratory of Power Transmission Equipment \& System Security and New Technology \\ Chongqing University, Shapingba District, Chongqing 400044, China \\ ${ }^{2}$ Tony Davis High Voltage Laboratory, School of Electronics and Computer Science \\ University of Southampton, Southampton SO17 1BJ, UK \\ ${ }^{3}$ Sichuan Electric Power Research Institute, State Grid, Chengdu, Sichuan 610072, China
}

\begin{abstract}
Partial Discharge (PD) is considered to be one of the main reasons for aging and degradation of the oil/pressboard insulation system in power transformers. Vegetable oils which own excellent dielectric performance are introduced as potential insulation liquids substituting traditional mineral oil. In this paper, an air-gap PD model was adopted to investigate the PD characteristics of refined rapeseed oil and Karamay 25\# mineral oil that are both currently employed in liquid filled power transformers. The PD current pulse waveform analysis (PD-CPWA) method was used to investigate PD mechanisms of two different insulation systems: mineral oil/pressboard and vegetable oil/pressboard. For both insulation systems, phase resolved partial discharge (PRPD) patterns throughout the accelerated deterioration experiments were compared. The extracted $\varphi-q-n$ plots with respect to various PD times were analyzed. It is found that, the air-gap PD stage characteristics of vegetable oil/pressboard are more notable. It has fewer double-peak pulses, smaller inception phase angle, lower charge amplitude, higher repetition rate, and more remarkable 'rabbit-ear' patterns than mineral oil/pressboard. The PD development process of both insulation systems can be characterized by four stages: initial discharge stage, weak developing stage, discharge burst stage and pre-breakdown stage.
\end{abstract}

Index Terms - Partial discharge, vegetable oil, mineral oil, characteristic analysis, oil/pressboard insulation, power transformer

\section{INTRODUCTION}

POWER transformers are one of the most critical pieces of apparatus in electrical power systems. Mineral oil is the most commonly used insulation liquid and coolant in transformers for its good dielectric and thermal properties [1]. However, with increasing concerns over environmental protection and fire safety, non-renewable, poorly biodegradable and low-flashpoint mineral oil is gradually being replaced by vegetable oils in transformers rated up to $420 \mathrm{kV}$. Based on natural ester oils, vegetable insulation oils are recyclable, have high biodegradability and are fire resistant [2,3]. Some studies have been carried out to investigate physical, dielectric and thermal characteristics of vegetable oils in recent years [4-10]. It was found that, refined vegetable oils can satisfy the requirements of dielectric fluid, however, studies on long gap breakdown of vegetable oils need to be carried out urgently for further application in transformers at or over $500 \mathrm{kV}$, and the design of

Manuscript received on X Month 2016, in final form XX Month 2016 , accepted XX Month 2016 large power transformers filled with vegetable oils should be carefully considered because of increased viscosity.

Partial discharge (PD) plays a significant role in causing the aging and deterioration of oil/pressboard insulation systems, and may ultimately lead to power transformer failures [11]. Air-gap discharge is one of the most common and serious PD defect types $[12,13]$. Air-gaps in solid dielectrics can result from many causes. In the case of transformer oil/pressboard insulation systems, for example, it can be caused by oil immersing into pressboard incompletely or air leaking into the gap between the winding and the pressboard. Although the magnitude of air-gap $\mathrm{PD}$ is relatively low, it can do serious harm to the solid insulation (pressboard) that cannot be self-recover [14,15]. Insulation breakdown in power transformers can cause serious accidents, bring about expensive maintenance or replacement costs and reduce the reliability of power supply. Thus, air-gap PD characteristics and development stages have become the focus of worldwide research.

However, although more than 1,000,000 transformers filled 
with vegetable insulation oils have been installed worldwide in the past decades, the PD characteristics and development process are still not clarified. Few reports on the PD behavior of vegetable oils have been made. It has been reported by Imamovic [16] and Wang [17] that biodegradable oil generates a higher level of dissolved gases than do mineral oil under partial discharge. Pompili [18] investigated PD pulse attributes of natural ester fluid using simultaneous wide and narrow band measurement techniques. Chandrasekar [19] collected PD patterns from thermally aged corn oil and palm oil. Nevertheless, most published studies only concentrated on discharge phenomena related to corona discharges and surface discharges, whilst air-gap PD mechanisms, characteristics of vegetable insulation oils during the whole development process have rarely been discussed.

It is now generally accepted that the PD current pulse waveform analysis (PD-CPWA) can be used to investigate PD mechanisms [20-24] and phase resolved partial discharge (PRPD) patterns can be used to assess the insulation status [2529]. Several modern mathematical analysis methods have also been introduced to process PD signals [30,31].

Given all of the above, the main purpose of this paper is to investigate typical air-gap PD mechanisms and characteristics of refined rapeseed oil/pressboard insulation systems during the whole development process from generating PD to breakdown (BD). These characteristics are then compared with those of traditional mineral oil/pressboard insulation system in order to provide a reference for recognizing air-gap PD development stages. The constant voltage pulsating current method is conducted according to the IEC standard 60270 [12], and the standard cylindrical air-gap PD model recommended by CIGRÉ [13] is adopted. PD current pulse waveforms, PRPD patterns and $\varphi$-q-n plots are collated with different PD times.

\section{EXPERIMENTAL DESCRIPTIONS}

\subsection{SAMPLE PREPARATION}

Two kinds of transformer insulation fluids were used for the experimental studies, namely, Karamay 25\# mineral oil (K125X, produced by China National Petroleum Corporation) and refined rapeseed oil (RDB, patents protected by Chongqing University). $\mathrm{K} 125 \mathrm{X}$, is a commercially used naphthenic oil, mainly consisting of naphthenes $(77 \%)$,

Table 1. Basic Properties of Two Types of Transformer Oil Specimens

\begin{tabular}{c|c|c|c}
\hline Property & Unit & RDB & $\mathrm{K} 125 \mathrm{X}$ \\
\hline Density at $20^{\circ} \mathrm{C}$ & $\mathrm{Kg} / \mathrm{dm}^{3}$ & 0.90 & 0.868 \\
Acid value & $\mathrm{mgKOH} / \mathrm{g}$ & 0.03 & 0.02 \\
Pour point & ${ }^{\circ} \mathrm{C}$ & -18 & -47 \\
Flash point & ${ }^{\circ} \mathrm{C}$ & 325 & 145 \\
Kinematic viscosity at $40^{\circ} \mathrm{C}$ & $\mathrm{mm}^{2} / \mathrm{s}$ & 43 & 10 \\
Biodegradability & $\%$ & 98 & 30 \\
Water content & $\mathrm{ppm}$ & $<200$ & $<20$ \\
AC breakdown & $\mathrm{kV}$ & 73 & 60 \\
Dissipation factor & $\%$ & 0.75 & 0.1 \\
Relative permittivity & & 3.2 & 2.2 \\
Volume resistivity at $90^{\circ} \mathrm{C}$ & $\Omega \mathrm{m}$ & $10^{11}$ & $10^{13}$ \\
\hline
\end{tabular}

paraffins $(11 \%)$ and aromatics $(10 \%)$, while RDB is a natural ester liquid, obtained from raw rapeseed oil using three main procedures (alkaline refinement, vacuum distillation and physical bleaching separately) $[7,32]$. The basic properties of the two dielectric fluids are shown in Table 1.

The liquid specimens and smoothly polished Kraft insulation pressboards (provided by Hunan No.1 Insulation Pressboard Co. Ltd, China) were treated in a vacuum oven at a temperature of $90{ }^{\circ} \mathrm{C}$ and pressure of $50 \mathrm{~Pa}$ for $48 \mathrm{~h}$ to dehydrate and degas. Then, fully dried pressboards were immersed in transformer oils under vacuum condition for a further $48 \mathrm{~h}$. After these procedures, the pressboard samples had been fully impregnated with transformer oils and cooled to ambient temperature. Table 2 shows the basic properties of dried pressboard, vegetable oil impregnated pressboard (VOIP) and mineral oil impregnated pressboard (MOIP).

Table 2. Basic Properties of Dried Pressboard, VOIP and MOIP

\begin{tabular}{c|c|c|c|c}
\hline Property & Unit & Dry & VOIP & MOIP \\
\hline Degree of polymerization & & 1091 & 1093 & 1085 \\
Density & $\mathrm{g} / \mathrm{cm}^{3}$ & 1.20 & 1.15 & 1.12 \\
Moisture content & $\%$ & 0.31 & 0.40 & 0.35 \\
AC breakdown & $\mathrm{kV}$ & 8.5 & 22.1 & 21.5 \\
Dissipation factor & $\%$ & 0.22 & 0.53 & 0.55 \\
Relative permittivity & & 4.75 & 4.11 & 3.68 \\
Volume resistivity at $90^{\circ} \mathrm{C}$ & $\Omega \mathrm{m}$ & $10^{11}$ & $10^{10}$ & $10^{11}$ \\
\hline
\end{tabular}

\subsection{PD EXPERIMENT SETUP}

The PD measurement circuit was designed and PD signals were detected according to the IEC standard 60270 [12], as depicted in Figure 1. A discharge-free AC voltage transformer $(60 \mathrm{kV} / 60 \mathrm{kVA})$ was utilized to stress samples at $50 \mathrm{~Hz}$. A $5.3 \mathrm{k} \Omega$ resistor was added between the transformer and test cell to avoid unexpected damage to electrodes and samples when breakdown occurred. A $1000 \mathrm{pF}$ coupling capacitor facilitated the passage of high-frequency current impulses. A wide-band digital oscilloscope (Lecroy Wavepro 7100, maximum $20 \mathrm{GS} / \mathrm{s}$ sampling rate and $2 \mathrm{GHz}$ bandwidth) was used to collect and store PD current pulse waveforms and PRPD patterns. A non-inductive $50 \Omega$ resistor, connected in parallel with the oscilloscope, was added to allow measurement of PD current pluses and protect the oscilloscope from damage. A $50 \mathrm{~Hz}$ phase voltage reference was obtained from high voltage divider, which was connected in parallel with the test cell.

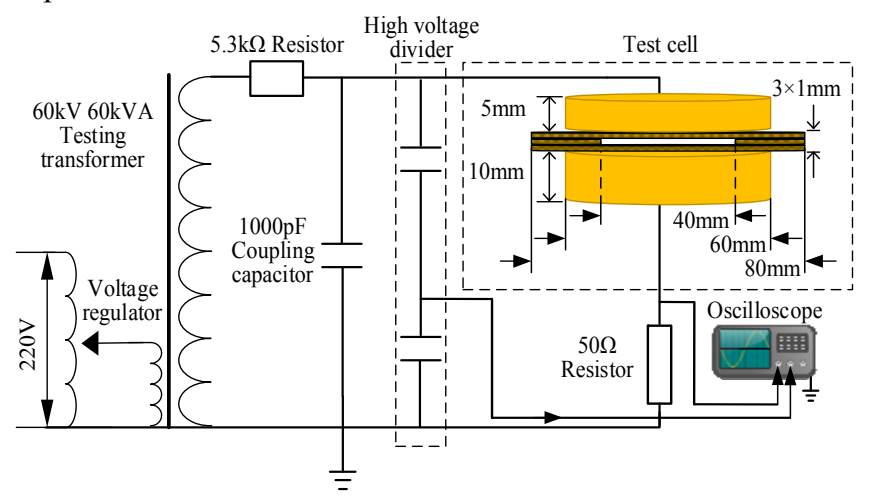

Figure 1. Schematic Circuit Diagram for PD Measurement 
The air-gap model was manufactured according to CIGRÉ Method II [13] and ASTM-D149-09 [33]. A cylindrical airgap was made by a ring of pressboard (40 mm internal diameter, $1 \mathrm{~mm}$ thickness) embedded between two pressboards, both with $80 \mathrm{~mm}$ diameter and $1 \mathrm{~mm}$ thickness. Insulating glue was employed to seal the air to avoid oil penetration during the PD experiment, and the initial pressure is $1 \mathrm{~atm}$. The pressboards were sandwiched between a pair of plane-plane brass electrodes. The upper plane electrode, with a diameter of $60 \mathrm{~mm}$ and a height of $5 \mathrm{~mm}$, was energized by high voltage during the test. The lower electrode, with the same diameter and a height of $10 \mathrm{~mm}$, was grounded. The edges of the electrodes were chamfered to prevent corona discharge. The test model was held in an oil tank, which was placed in a thermal-aging chamber at a predefined temperature of $60{ }^{\circ} \mathrm{C}$ during the test. An oil circulating system was designed and the flow velocity was set to $0.8 \mathrm{~L} / \mathrm{min}$. The experimental apparatus is the same as the one mentioned in relevant literature [34].

\subsection{PD EXPERIMENT PROCEDURE}

Prior to tests, the apparent charge was modified using a correction circuit in accordance with to the IEC standard 60270 [12]. The relationship between apparent discharge and pulse voltage was expressed in the terms of following equation. It was also verified that the background noise did not exceed $5 \mathrm{pC}$ up to $60 \mathrm{kV}$.

$$
q_{0}=0.4978 U+0.084
$$

The first step of the experiment is to determine the PD inception voltage (PDIV). The supply voltage was increased slowly in steps of $0.5 \mathrm{kV} / 10 \mathrm{~s}$, up to the level at which the magnitude of apparent discharge reached $100 \mathrm{pC}$ (according to IEC 61294) [35]. Each test was repeated 10 times to obtain the mean value of PDIV. It was found that vegetable oil has a slightly lower PDIV than mineral oil, $7.2 \mathrm{kV}$ and $8.1 \mathrm{kV}$ respectively; similar results are shown in [18]. Generally, $120 \%-140 \%$ of PDIV should be chosen as the test voltage to observe more representative PD behavior. To satisfy this condition, the applied voltage was manually ramped to $10 \mathrm{kV}$ at a rate of $500 \mathrm{~V} / 30 \mathrm{~min}$, and then overstressed for $2 \mathrm{~h}$ in both insulation systems in order to reach a quasi-static condition and compare PD characteristics.

During the whole PD process, the bandwidth of the digital oscilloscope was set to $1 \mathrm{GHz}$. The PD current pulses were obtained at a sampling rate of $2 \mathrm{GS} / \mathrm{s}$ and PRPD patterns were captured at a sampling rate of $10 \mathrm{MS} / \mathrm{s}$ for 500 cycles $(10 \mathrm{~s})$ every $30 \mathrm{~min}$. Each point in the PRPD pattern represents one PD pulse.

\section{EXPERIMENTAL RESULTS AND DISCUSSION}

Due to the disparate molecular components of mineral oil and vegetable oil, the dielectric and thermal properties of mineral oil/pressboard and vegetable oil/pressboard are diverse. Under continuous AC applied voltage, the two insulation systems will show various PD characteristics in small air-gaps.

The PD current pulse waveform analysis (PD-CPWA) is a commonly accepted method of analyzing PD mechanisms, especially for $\mathrm{SF}_{6}$ gas. PRPD patterns and $\varphi$-q-n plots are widely used to describe the characteristics of PD. In this section, typical current pulse waveforms at various air-gap discharge-times are recorded and PD mechanisms of different oil/pressboard insulation systems are discussed. These data provide a basis for the following explanation of the different PRPD patterns and $\varphi$-q-n plots observed.

\subsection{PD CURRENT PULSE WAVEFORMS}

Figure 2 illustrates the definition of PD parameters on a current pulse waveform, such as rise time $t_{r}, d i / d t\left(\simeq I / t_{r}\right)$, peak current $I$ and fall time $t_{f} . t_{r}$ is related to electron avalanche formation and streamer propagation during PD extension; $d i / d t$ reflects the generation rate of effective electrons, which corresponds to the velocity of $\mathrm{PD}$ extension; $t_{f}$ refers to electron drift in the air-gap where PD occurs [21-24].

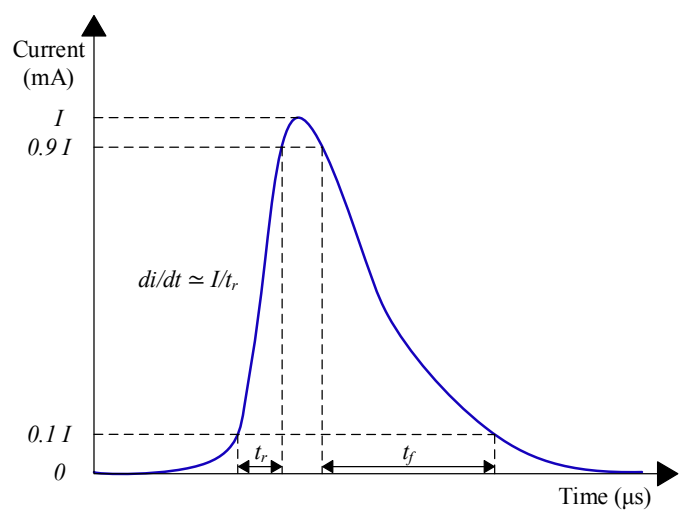

Figure 2. Definition of PD Parameters

Typical measured PD current pulse waveforms at different PD times are shown in Table 3. It can be seen that, at the beginning of PD, both insulation systems show standard pulse shape and the amplitudes are small. During the fall time, $t_{f}$, small bulges appear and become more obvious with the variation of PD time due to the increasing number of free electrons provided by the PD pulses.

Because of the degradation of oil and pressboard, electronegative gases are released into the air-gap gradually, such as $\mathrm{CO}, \mathrm{CO}_{2}$ and $\mathrm{O}_{2}$. These gases can attach free electrons generated by the preceding PD pulse, leading to an increase in time-lag to the next pulse [36]. Therefore, an over-voltage is applied on the sample, causing an increase in current magnitude. Moreover, the second PD pulse occurs in the middle of the fall time, with a relatively smaller magnitude. The reason is that the first PD pulse provides free electrons, which can act as a trigger to form another electron avalanche under a much lower over-voltage for shorter time-lag. As a result, the double-peak current pulses appear due to the influence of electronegative gases. 
As the PD process develops, current pulses of much larger magnitude are detected in both insulation systems. This is due to the continuous deterioration of insulation materials under PD stress and more electrons attached by the increasing concentration of electronegative gases. However, it was verified that the generation rate of double-peak current pulses decreases when the gas pressure attains a high level at a constant applied voltage. This is because highly electronegative gases suppress the formation of electron avalanches [21-23].

The main components of mineral oil are naphthenes, paraffins, and aromatics, presenting very weak polarity for symmetrical structures, while vegetable oil consists primarily of triglycerides, which are polar molecules due to generally different fatty acid groups. The chemical structures of the main components in the two dielectric liquids are shown in Table 4. Consequently, the relative permittivity of vegetable oil is closer to that of insulation pressboard and the relative permittivity of VOIP is larger than MOIP, as shown in Table 1 and Table 2 separately. Since the conductivity of air-gap and oil impregnated pressboard is so small under ac stress that it can be ignored, the electric field intensity ratio of air-gap to oil impregnated pressboard can be defined as:

$$
\frac{E_{c}}{E_{b}}=\frac{u_{c} / \delta}{u_{b} /(d-\delta)}=\frac{\varepsilon_{b}}{\varepsilon_{c}}
$$

where $\varepsilon_{c}$ and $\varepsilon_{b}$ represent the relative permittivity of air-gap and oil impregnated pressboard respectively. Because of the influence of the polarity of $\mathrm{RDB}$, the larger relative permittivity of VOIP makes the air-gap withstand a higher electric field intensity. Besides, it is clear to see that vegetable oil contains oxygen element in its chemical structure while mineral oil does not, leading to increased generation of electronegative gases when the dielectric liquid is decomposed
Table 4. Chemical Structures of Main Compounds in Transformer Oils

\begin{tabular}{|c|c|c|c|c|}
\hline Compound & Naphthene & Paraffin & Aromatic & Triglyceride \\
\hline Structure & & $\begin{array}{ccc}\mathrm{H} & \mathrm{H} & \mathrm{H} \\
\mathrm{I} & \mathrm{I} & 1 \\
\mathrm{C} & -\mathrm{C} & - \\
\mathrm{l} & \mathrm{C} & \mathrm{I} \\
\mathrm{H} & \mathrm{H} & \mathrm{H}\end{array}$ & & $\begin{array}{l}\mathrm{CH}_{2}-\mathrm{OOCR}_{1} \\
\mathbf{I} \\
\mathrm{CH}-\mathrm{OOCR} \\
\mathbf{1} \\
\mathrm{CH}_{2}-\mathrm{OOCR}_{3}\end{array}$ \\
\hline
\end{tabular}

under higher electrical stress, especially for $\mathrm{O}_{2}, \mathrm{CO}$ and $\mathrm{CO}_{2}$ [17].

In consequence, the two insulation systems show distinctive characteristics. The electron avalanche of subsequent PD pulse in vegetable oil/pressboard insulation system is suppressed by high gas content and, thus, the PD current pulse waveform returns to its original shape. In contrast, more peaks are triggered simultaneously in the typical PD current pulse waveform of mineral oil/pressboard insulation system because of the low gas content and also because amounts of free electrons are generated from the first PD pulse which has extremely high magnitude. Current pulses with more peaks can transfer much more PD charge, which can be calculated by integrating the area under the current-time curve. Therefore, PD charge is accumulated more rapidly in the $\mathrm{K} 125 \mathrm{X}$ system and more PD energy is generated compared to $\mathrm{RDB}$, and this does greater harm to the deterioration of insulation pressboards.

In the presence of vast fault gases, subsequent peaks in current are gradually separated, whereas several PD pulses with small magnitude appear at the end of the first PD pulse instead. Similar current pulses lie in vegetable oil/pressboard before $\mathrm{BD}$, which indicate that the small PD pluses play an important role in extending the BD. A similar conclusion was drawn by Okubo for thermal degraded epoxy resin [21]. Although the height of PD current pulse is smaller than the

Table 3. Typical PD Current Pulse Waveforms of Oil/pressboard During Air-gap PD Process
Sample
(a) 1 hours
(b) 10 hours
(c) 18 hours
(d) Pre-BD

K125X
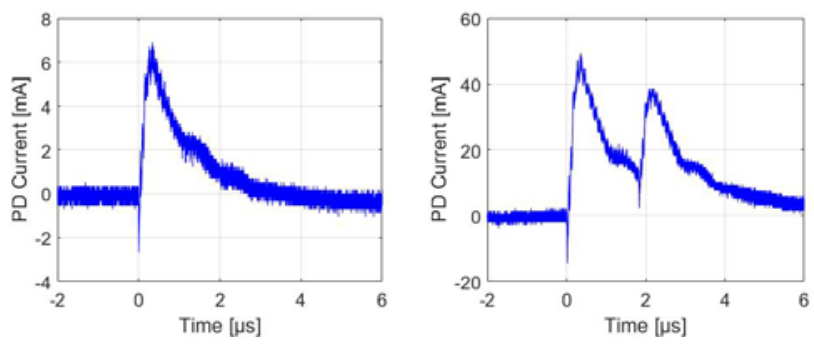

RDB

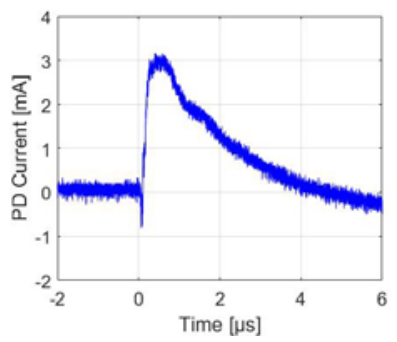

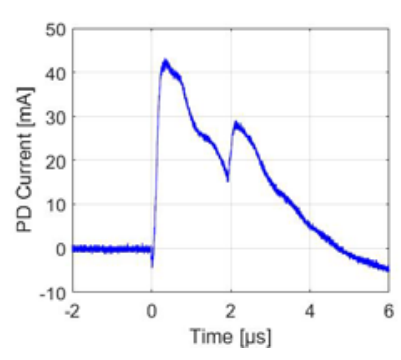

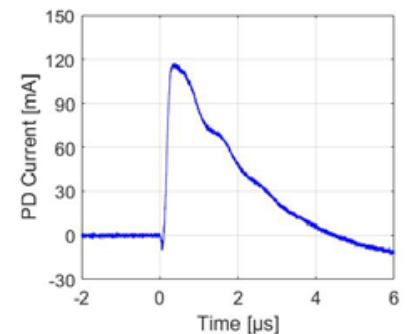

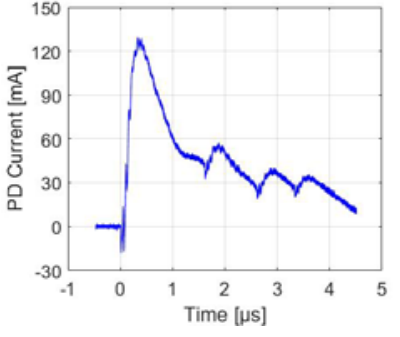
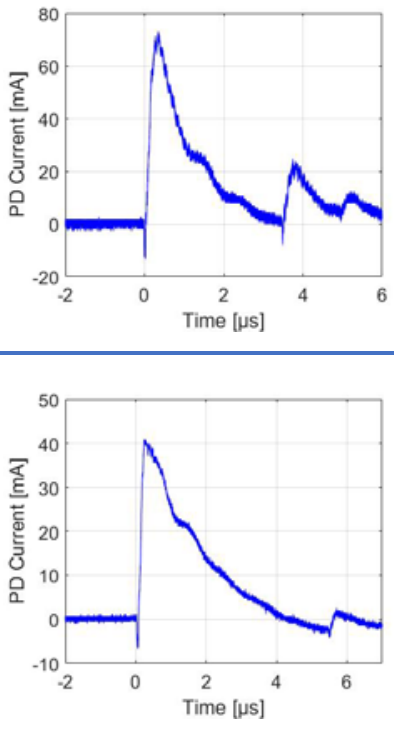

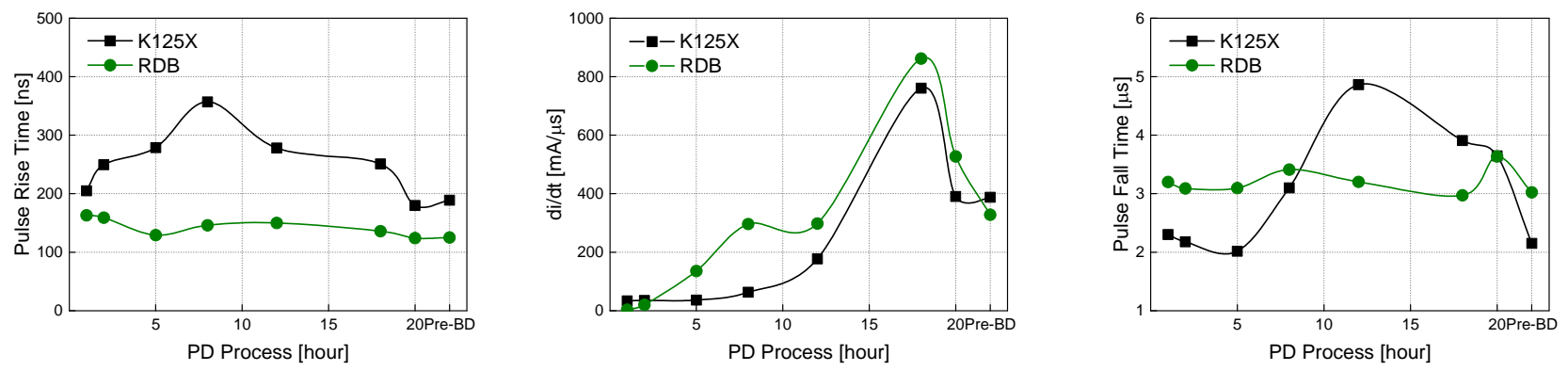

last period, it still can lead to BD for the badly damage of insulation pressboards. Because the development of air-gap PD mainly depends on the actual stress in the air-gap defect and the deterioration of oil impregnated pressboard, higher stress in the air-gap of RDB and faster deterioration of mineral oil impregnated pressboard contribute to the similar time frames of these two systems.

Table 5 shows the variation of PD parameters with different PD times, rise time, $t_{r}$, di/dt and fall time, $t_{f}$, respectively. In the PD-CPWA, the variation in PD-parameters with time can be used to investigate the long-time PD generation characteristics.

The first free electron must be generated for triggering electron avalanche to form a PD pulse [37]. The rise time, $t_{r}$, is determined by the movement of electrons from the cathode towards the anode, with a time of flight of the order of nanoseconds. When PD occurs, the electron hits the insulation pressboard with high speed and this breaks apart the chemical bonds in the cellulose surface, leading to the generation of $\mathrm{CO}$ and $\mathrm{CO}_{2}$. In the mineral oil/pressboard insulation system $\mathrm{K} 125 \mathrm{X}, t_{r}$ rises dramatically with more free electrons attached by electronegative gases generated from the deterioration of the pressboard. This suggests that electron avalanche and streamer propagation are more active and the PD extension is longer. PD activity increases after double-peak current pulses appear and more fault gases are generated. Higher gas content has a negative effect on the electron avalanche process; however, $t_{r}$ still remains high because of the low generation rate of gases. The concentration of fault gases is greatly increased at the end of the PD process due to cumulative insulation deterioration, which suppresses electron avalanche and cause $t_{r}$ to decrease to a much lower level [23]. In the vegetable oil/pressboard insulation system RDB, the generation rate of electronegative gases is much higher, leading to shorter duration of the double-peak-pulse phenomenon. As a consequence, $t_{r}$ remains more stable in the range 100-200 ns, whilst the developing tendency of $t_{r}$ is similar to that of $\mathrm{K} 125 \mathrm{X}$.

The development of electron avalanches depends on the electron impact ionization process $\alpha$ and attachment $\eta$ [24]. The gradient of effective ionization coefficient $\alpha_{g r a}$ can be defined by the formula

$$
\alpha_{\text {gra }}=\left(\frac{\alpha-\eta}{P}\right)\left(\frac{E_{c r}}{P}\right)^{-1}
$$

where $E_{c r}$ is the critical electric field strength and $P$ is the pressure in the air-gap. $d i / d t$ is found to have a positive correlation with $\alpha_{g r a}$ of electronegative gases, which reflects the electric field sensitivity of the ionization activity [21]. As shown in Table 5, RDB exhibits a similar trend to K125X, with a rapid rise in the development of $\mathrm{PD}$ and subsequent decrease prior to $\mathrm{BD}$. The value of $d i / d t$ is higher generally. For RDB meaning that effective electrons are generated more quickly in the air-gap of vegetable oil/pressboard than mineral oil/pressboard. Furthermore, the change in PD current pulse peak can be attributed to the variation of $d i / d t$, which is related to streamer propagation.

The mechanism of fall time $t_{f}$ can be explained with reference to the physical characteristics of electron drift after the formation of an electron avalanche. Concerning the $\mathrm{K} 125 \mathrm{X}$ system, $t_{f}$ remains relatively stable at a lower level at the beginning until the presence of double-peak PD current pulses, which leads to a dramatic rise in the PD duration. When the gas content in the air-gap exceeds a critical value, electronegative gases begin to suppress electron avalanche formation, resulting in a reduction in the number of electrons. Besides, more electrons in the air-gap disappear due to the increase in electronegativity. Therefore, the fall time, $t_{f}$, shows declining trend. However, the suppression effect on $t_{f}$ can be ignored until the gas content is high enough to separate the subsequent pulses from the first PD current pulse. In the RDB system, $t_{f}$ exhibits a similar trend, with slight fluctuation in the range of $3 \mu \mathrm{s}$, also because of the high generation rate of electronegative gases.

Therefore, PD-CPWA can be used to investigate air-gap PD mechanisms of insulation oil/pressboard systems and analyze the differences in individual PD current pulses of K125X and RDB. Moreover, the deterioration of insulation systems and the PD development process can be estimated by analyzing PD parameters. 


\subsection{PRPD PATTERNS}

Table 6 shows typical PRPD patterns obtained from vegetable oil/pressboard insulation system and mineral oil/pressboard insulation system after various PD times.

At the beginning of PD in $\mathrm{K} 125 \mathrm{X}$, the initial discharge signals only occur around the peak of the negative half cycle with small magnitude. With the development of PD, discharge signals are detected in the positive half cycle and grow rapidly. The PD magnitude increases and the phase range expands gradually in the following $20 \mathrm{~h}$, with the phase angle corresponding to the maximum PD amplitudes slowly shifting forward from $270^{\circ}$. During the period of pre-breakdown (Pre$\mathrm{BD})$, the bulk of the large magnitude PD pulses concentrate in the positive half cycle over a wide phase range, while only small amplitudes are detected in the negative half cycle.

With regard to $\mathrm{RDB}$, the first $\mathrm{PD}$ also occurs in the negative half cycle at relatively smaller phase angles, and few small pulses in the positive half cycle appear earlier. The evolution process of the PRPD patterns (increasing magnitude and widening phase range) is similar. However, the inception phase expands more significantly, and the PD pulses can even occur at $0^{\circ}$ in the positive half cycle and $180^{\circ}$ in the negative half cycle just prior to BD. Therefore, the shift of PD occurrence phase angle need to be well discussed in this section.

As mentioned above, the initial PD signal tends to occur more often at the peak voltage value of the applied sine wave. Similar phenomena in insulation systems have been recorded in previous studies $[38,39]$, which shows that the electric field plays a major role in providing enough energy for PD formation. However, even if the critical electric field is exceeded, the first free electron may not immediately trigger an avalanche, since a neutral gas molecule may absorb the electron and becoming a negative ion or the kinetic energy of electron may not be enough to initiate ionization. Hence, a statistical time lag appears before PD [29]. The first electron is mainly generated by cathode emission in the negative half cycle, whilst the initial electron in the positive half cycle comes from volume ionization in the air-gap. In the air-gap that has never experienced PD, negative ions and energetic photons are too scarce to generate effective electrons and the low temperature restricts the process of thermal ionization. In contrast, electron tends to be emitted from the cathode more easily. Thus, the first electron might be generated by cathode emission, such that the first PD pulse often appears in the negative half cycle. This phenomenon is consistent after repeated testing.

After the first PD pulse is generated, the electrons pass through air-gap and hit the pressboard at high velocity. Various characteristics of different oil/pressboard insulation systems are shown at the beginning of PD. It is worth noting that the relative permittivity of vegetable oil-impregnated pressboard is higher than that of mineral oil-impregnated pressboard, which leads to relatively larger amorphous regions and smaller crystal regions under the stress of electric field. In crystal region, molecule chains of cellulose are arranged an orderly and compact configuration, resulting in a low degree of freedom and weak flexibility, under the influence of hydrogen bonds and Van der Waals force. Conversely, in amorphous areas, the molecule chains of cellulose are arranged randomly. This brings about a high degree of freedom and flexibility. When the electrons hit the surface of pressboard with high speed, the molecule chains in crystal region are broken up more easily, leading to much more severe damage than in amorphous area [40]. Therefore, considering the two reasons above, more shallow traps are formed on the surface of vegetable oil-impregnated pressboard, however, the number of deeper traps is growing on the surface of mineral oil-impregnated pressboard under PD, which causes more electrons to be captured.

Table 6. Typical PRPD Patterns of Oil/pressboard During Air-gap PD Process
Sample
(a) 1 hours
(b) 8 hours
(c) 18 hours
(d) Pre-BD

$\mathrm{K} 125 \mathrm{X}$
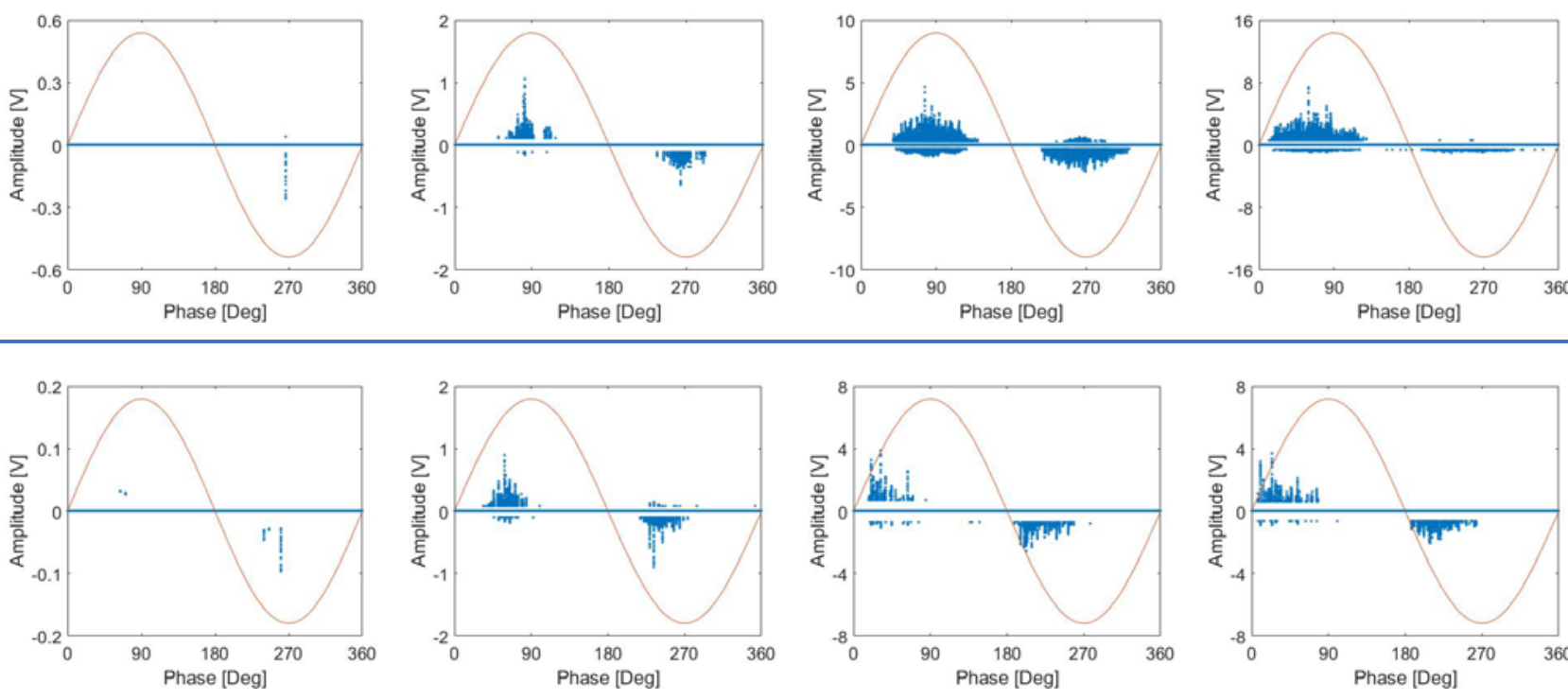
After PD occurs in the air-gap, almost all new electrons, which start the next electron avalanche, come from the electrons which were left on the air-gap wall by the preceding discharge. The electron generation rate basically depends on the surface emission $P_{\text {surf }}$, which is defined by the RichardsonSchottky equation [41]

$$
P_{\text {surf }}(t)=N_{s c}(t) v_{o} \exp \left[-\frac{\psi-\sqrt{q^{3}\left|E(t) /\left(4 \pi \varepsilon_{0}\right)\right|}}{K T}\right]
$$

where $N_{s c}(\mathrm{t})$ is the number of electrons trapped by the air-gap wall; $\psi$ is the detrapping work function and $T$ is the temperature in air-gap. The average statistical time lag is inversely proportional to the electron generation rate. According to the empirical formula, the average statistical time lag of cylindrical air-gap can be calculated using

$$
\overline{t_{0}}=\frac{1}{C_{r a d} \Phi_{r a d}(\rho / p)_{0} P_{\text {surf }}(t) \pi r 2\left(h-l_{\text {inc }}\right)}
$$

In the air-gap of mineral oil/pressboard system, the high-speed electrons tend to be captured by the deeper traps, leading to a high detrapping work function. When the polarity of applied voltage changes to the positive half cycle, the electrons cannot get enough energy to be emitted from the traps to initiate a further electron avalanche. Therefore, few PD signals are detected in the positive half cycle at the beginning of PD. With the development of PD, the amount of electrons increases and the pressboard is gradually degraded. Consequently, some electrons kept in relatively shallower traps are triggered under higher energy to form electron avalanches. This leads to PD signals appearing in the positive half cycle. Even though the generation rate of electrons rises slightly and the PD occurrence phase angle shifts forward, the phenomenon is not obvious. The reason is that, higher velocity of electrons and thermal effect of PD bring about severe damage of pressboard with increasing number of deep traps on the surface, and the detrapping work function is still kept at a high level. During the period of Pre-BD, the PD phase angle shifts remarkably due to increased numbers of shallow traps [42].

In the air-gap of vegetable oil/pressboard, when PD occurs, some electrons are kept in shallow traps, and can be emitted easily from the surface of pressboard under relatively lower voltage. Higher $N_{s c}(\mathrm{t})$ and lower $\psi$ result in higher $P_{\text {surf }}$, shorter statistical time lag and smaller PD occurrence phase angle. As a result, some signals can be detected earlier in the positive half cycle and the regular PD pulses occur over the ascending portions, which are near to the peak of the applied sinusoidal voltage. However, due to the low number of electrons, these PD signals are quite weak. With the development of PD, the amount of electrons, which might bring about more shallow traps, is increasing. Furthermore, it is noteworthy that the temperature of the discharging point may exceed $1000{ }^{\circ} \mathrm{C}$ [40], while vegetable oil/pressboard presents higher thermal stability than mineral oil/pressboard [43]. More shallow traps tend to be generated on the surface of vegetable oil/pressboard instead of deep traps with less influence of high temperature. Therefore, the increasing number of shallow traps and detrapping electrons during the PD development process lead to the continuous decrease in PD occurrence phase angle, which shows more notable PD characteristics of RDB than that of $\mathrm{K} 125 \mathrm{X}$.

\subsection{PD Ф-Q-N PLOTS}

Table 7 shows PD $\varphi$-q-n plots obtained from the mineral oil/pressboard insulation system and vegetable oil/pressboard insulation system increasing PD times, which provide a clear description of the variations of PD charge magnitude and PD pulse number with respect to phase angle.

In the air-gap of mineral oil/pressboard, it can be perceived that the number of PD pulses with high charge magnitude increases significantly, whilst, low charge amplitudes maintain

Table 7. $\varphi-q-n$ Plots of Oil/pressboard During Air-gap PD Process
Sample
(a) 1 hours
(b) 8 hours
(c) 18 hours
(d) Pre-BD

K125X

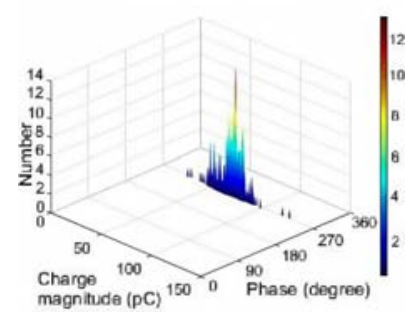

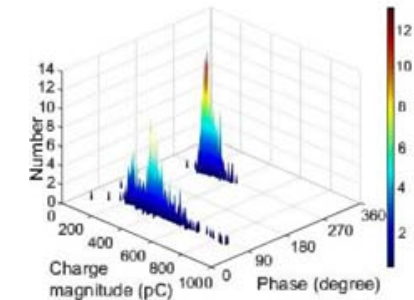

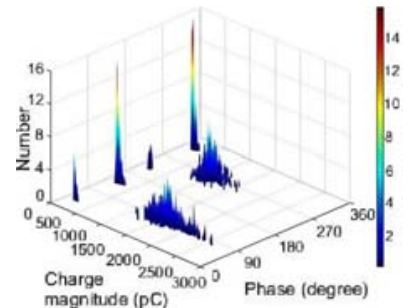

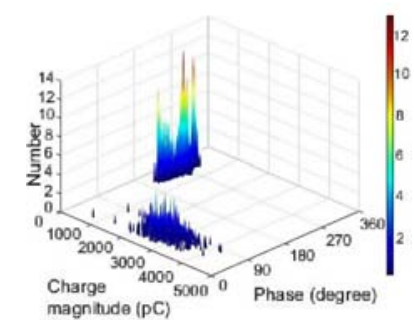

RDB
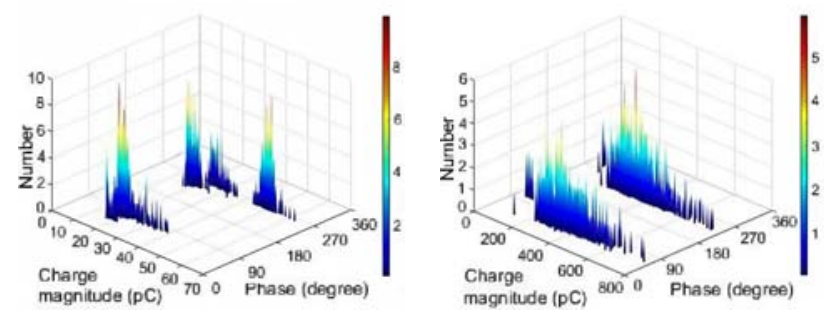

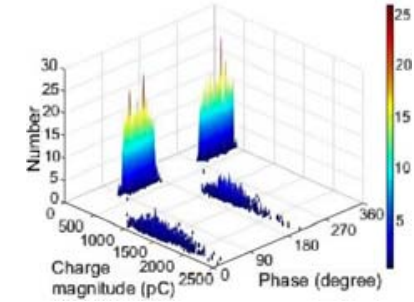


a relatively higher repetition rate. Moreover, the number of PD pulses in the negative half cycle is generally larger than the number of pulses in the positive half cycle. In comparison, similar developing tendency of PD signals is depicted in vegetable oil/pressboard, however, the charge magnitude is lower and the repetition rate is higher generally, especially at the end of PD. Similar results are observed when testing on other types of vegetable oils under PD faults $[18,19]$.

The difference in charge magnitude of the two insulation systems can be well explained by the double-peak PD current pulse phenomenon mentioned in Section 3.1. The similarity of the two insulation systems is that the charge magnitude in the positive half cycle is generally higher than that in the negative half cycle, which results in the asymmetry in these figures. This phenomenon is due to the number of charges captured by deep traps on the surface of the lower pressboard is generally larger than that of the upper pressboard. When the polarity of the applied voltage changes to the positive half cycle and an over-voltage is applied, these charges could be released. Thus, the charge magnitudes would be larger and the PD signals reach a relatively higher voltage amplitude, as shown in Table 6. Moreover, because of the larger number of deep traps on the surface of mineral oil impregnated pressboard, the asymmetry of charge magnitude is more obvious in the figures relating to the $\mathrm{K} 125 \mathrm{X}$ system.

As for repetition rate in one cycle, $n$, it can be calculated using

$$
n=\frac{4 U_{m}}{U_{c}-U_{r}} \cdot \frac{C_{b}}{C_{b}-C_{c}}
$$

where $U_{m}$ is the magnitude of the applied voltage; $U_{c}$ is the critical inception voltage of $\mathrm{PD} ; U_{r}$ is the residual voltage caused by the preceding PD pulse, which can be defined as

$$
U_{r}=\gamma(E / p)_{c r}(p a)
$$

where $\gamma$ is the ratio of streamer path voltage to critical voltage, depending on gas type and polarity of streamer. In the air-gap of pressboard, this ratio for negative streamers is larger than for positive streamers, being approximate 0.5 and 0.2 respectively, leading to higher residual voltage. Therefore, the repetition ratio of $\mathrm{PD}$ pulses in the negative half cycle is larger than in the positive half cycle. With the development of PD, the PD occurrence phase angles shift in RDB more obviously than in K125X, which brings about a lower critical inception voltage and, thus, RDB shows a higher repetition rate than K125X.

The 'rabbit-ear' pattern has been investigated as a typical pattern of air-gap PD [29,44]. This phenomenon can be described that after a change in polarity of applied voltage, the first PD pulse occur with high charge magnitude, followed by the subsequent PD pulses with much lower magnitude. Prior studies show that water, oxygen and other electronegative gases play crucial roles in forming such a pattern [20]. Timelag is increased due to the attachment of electrons, leading to the enhancement of over-voltage and higher magnitude, which is the 'ear' part of the 'rabbit-ear' pattern. The subsequent PD pulses, with higher repetition rate and much lower magnitude due to the decrease in time-lag and low over-voltage, form the

\section{'body' part.}

As shown in Table 7, the 'rabbit-ear' patterns are more marked in vegetable oil/pressboard insulation system. At the beginning of $\mathrm{PD}$, oxygen and water are consumed as a consequence of oxidative activity, whilst free radical of oxygen, carbon oxides and water generated by radiation and thermal effects of PD are scarce, and hence, the 'rabbit-ear' pattern is not apparent. It has been pointed out in Section 3.1 that, with the increase in PD time, electronegative gases are generated in the vegetable oil/pressboard insulation system. More importantly, water is released by heating and bombardment during PD degradation and the water absorption characteristics of the vegetable oil influences the water content in the air-gap of vegetable oil/pressboard. Water is more likely to be absorbed by vegetable oil instead of cellulose and triglycerides tend to be decomposed to glycerol and fatty acids in the presence of moisture, leading to the occurrence of esterification reactions between active hydroxyls of cellulose and fatty acids. Long alkyl chains in fatty acids with hydrophobic characteristic are arranged in nearly parallel with cellulose, which leads to a 'water barrier' on the surface of cellulose, avoiding extensive water penetration into the pressboard [6]. Chemical reactions are presented as equations $(8,9)$. In contrast, because of the low polarity of mineral oil, the mutual attraction between water and cellulose is relatively much stronger and, thus, more water tends to be absorbed by the cellulose, leading to further deterioration of the pressboard. Therefore, based on the explanations given above, the water content in the air-gap of the vegetable oil/pressboard is higher than for the mineral oil/pressboard. High contents of water and electronegative gases contribute to the generation of the 'rabbit-ear' patterns, and their 'ears' rise vertically to the phase axis. Moreover, it was investigated that surface conductivity can be inferred as a factor in the formation of 'rabbit-ear' patterns [28], while vegetable oil shows higher conductivity, leading to more evidence of this phenomenon. Consequently, more remarkable 'rabbit-ear' patterns reflect more striking PD characteristic of RDB than that of K125X.
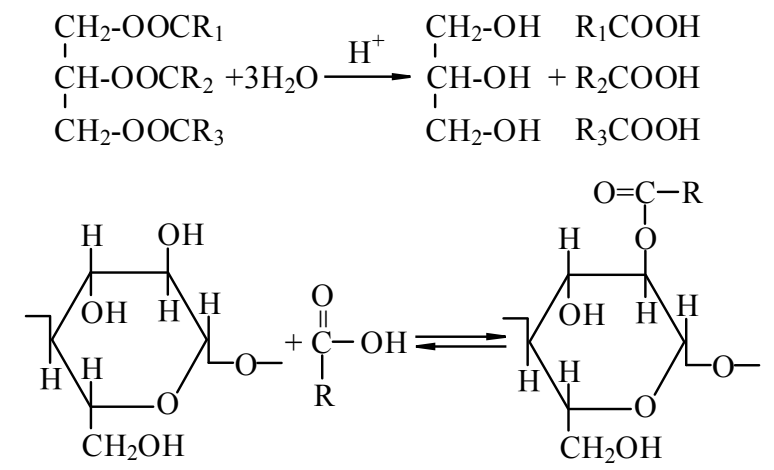

To conclude, the differences in the thermal, electrical and chemical properties of the two insulation systems determine the patterns. High magnitude of charges and severe damage of pressboard causing by high temperature and high-energy electrons indicate the end of mineral oil/pressboard insulation life, whilst high repetition of PD pulse plays a critical role in 
causing BD of vegetable oil/pressboard insulation system.

\section{CONCLUSION}

In this paper, laboratory experiments on a vegetable oil/pressboard insulation system (RDB) and a mineral oil/pressboard insulation system (K125X) under air-gap PD were carried out to investigate PD characteristics. The PDCPWA method was used to investigate diverse PD mechanisms, while the PRPD patterns and the $\varphi-q-n$ plots depict significant differences in phase angles, charge magnitude and repetition rate. More obvious decrease of PD occurrence phase angle and more remarkable 'rabbit-ear' patterns during the PD development process indicate that the air-gap PD characteristics of RDB are more notable than K125X, which offers great help to recognize PD development process in this system. Comparative conclusions of PD characteristics can be summarized in four different stages:

(1) During the initial discharge stage, both insulation systems show similar shape of PD current pulses, charge magnitude and repetition rate. Slight differences are that the RDB is characterized by smaller inception discharge phase angle and PD pulses in positive half cycle are detected earlier, due to the influence of the more polar character vegetable oils.

(2) In the weak developing stage, double-peak PD current pulses appear and the charge magnitude increases in both insulation systems. However, care should be taken for the shift of PD occurrence phase angle in RDB, which is much more marked, due to more shallow traps and higher generation rate of effective electrons.

(3) In the discharge burst stage, the magnitude of the PD pulses of two insulation systems shows a dramatic increase. However, special attention should be paid to the shape of the PD current pulses. More peaks are triggered in $\mathrm{K} 125 \mathrm{X}$ owing to much lower gas pressure and fewer electronegative gases in the air-gap, leading to increased space charges. Moreover, the 'rabbit-ear' patterns are more apparent in $\mathrm{RDB}$, and the main reason is that chemical reactions between vegetable oil and cellulose bring about higher water content in the air-gap.

(4) In the pre-breakdown stage, the first PD current pulse, which occurs in the air-gap, is followed by small PD current pulses in both systems, which can be reckoned as a signal of the final BD of the whole system. Furthermore, K125X presents higher charge magnitude, whilst the distinctive feature of $\mathrm{RDB}$ is higher repetition rate.

\section{ACKNOWLEDGMENT}

We would like to acknowledge the financial support by Science and Technology Project of China Southern Power Grid (GZGD20130301240157). One of the authors (Lu Cui) wishes to thank the Chinese Scholarship Council Funding for Joint Training Ph.D. Student (201506050096).

\section{REFERENCES}

[1] T. O. Rouse, "Mineral insulating oil in transformers", IEEE Electr. Insul. Mag., Vol. 14, No.3, pp. 6-16, 1998.

[2] T. V. Oommen, "Vegetable oils for liquid-filled transformers", IEEE Electr. Insul. Mag., Vol. 18, No.1, pp. 6-11, 2002.

[3] CIGRÉ working group A2.35: "Technical brochure 436 - experiences in service with new insulating liquids", 2010.

[4] D. Martin, N. Lelekakis, W. Guo, and Y. Odarenko, "Further studies of a vegetable-oil-filled power transformer", IEEE Electr. Insul. Mag., Vol. 27, No.5, pp. 6-13, 2011.

[5] I. L. Hosier, A. Guushaa, E. W. Westenbrink, C. Rogers, A. S. Vaughan, and S. G. Swingler, "Aging of biodegradable oils and assessment of their suitability for high voltage applications", IEEE Trans. Dielectr. Electr. Insul., Vol. 18, No.3, pp. 728-738, 2011.

[6] L. Yang, R. Liao, C. Sun, and M. Zhu, "Influence of vegetable oil on the thermal aging of transformer paper and its mechanism", IEEE Trans. Dielectr. Electr. Insul., Vol. 18, No.3, pp. 692-700, 2011.

[7] J. Li, Z. Zhang, P. Zou, S. Grzybowski, and M. Zahn, "Preparation of a vegetable oil-based nanofluid and investigation of its breakdown and dielectric properties", IEEE Electr. Insul. Mag., Vol. 28, No.5, pp. 4350, 2012 .

[8] Y. Xu, S. Qian, Q. Liu, and Z. Wang, "Oxidation stability assessment of a vegetable transformer oil under thermal aging", IEEE Trans. Dielectr. Electr. Insul., Vol. 21, No.2, pp. 683-692, 2014.

[9] H. M. Wilhelm, V. Franch, L. Tulio, and A. F. Franch, "Compatibility of transformer construction materials with natural ester-based insulating fluids", IEEE Trans. Dielectr. Electr. Insul., Vol. 22, No.5, pp. 27032708, 2015.

[10] L. Loiselle, I. Fofana, J. Sabau, S. Magdaleno-Adame, and J. C. Olivares-Galvan, "Comparative studies of the stability of various fluids under electrical discharge and thermal stresses", IEEE Trans. Dielectr. Electr. Insul., Vol. 22, No.5, pp. 2491-2499, 2015.

[11] T. K Saha, "Review of modern diagnostic techniques for assessing insulation condition in aged transformers", IEEE Trans. Dielectr. Electr. Insul., Vol. 10, No.5, pp. 903-917, 2003.

[12] IEC 60270: "High voltage test techniques - partial discharge measurement", 2000.

[13] CIGRÉ working group D1.33: "Guide for partial discharge measurements in compliance to IEC 60270", 2008.

[14] S. A. Boggs, "Partial discharge. III. Cavity-induced PD in solid dielectrics”, IEEE Electr. Insul. Mag., Vol. 6, No.6, pp. 11-16, 1990.

[15] G. C. Montanari and A. Cavallini, "Partial discharge diagnostics: from apparatus monitoring to smart grid assessment," IEEE Electr. Insul. Mag., vol. 29, pp. 8-17, 2013.

[16] D. Imamovic, K. X. Lai, N. A. Muhamad, B. T. Phung, and T. R. Blackburn, "Partial discharge and dissolved gas analysis in biodegradable transformer oil", Proc. CIGRÉ 2007 Colloquium, pp. 1-7, 2007.

[17] Z. D. Wang, X, Wang, X, Yi, S, Li, and J. V. Hinshaw, "Gas generation in natural ester and mineral oil under partial discharge and sparking faults”, IEEE Electr. Insul. Mag., Vol. 29, No.5, pp. 62-70, 2013.

[18] M. Pompili, C. Mazzetti, and R. Bartnikas, "Comparative PD pulse burst characteristics of transformer type natural and synthetic ester fluids and mineral oils", IEEE Trans. Dielectr. Electr. Insul., Vol. 6, No.16, pp. 1511-1518, 2009.

[19] S. Chandrasekar and G. Montanari, "Analysis of partial discharge characteristics of natural esters as dielectric fluid for electric power apparatus applications", IEEE Trans. Dielectr. Electr. Insul., Vol. 21, No.3, pp. 1251-1259, 2014.

[20] H. Ichikawa, K. Ito, Y. Suzuoki, T. Mizutani, and K. Uchida, "Partial discharge patterns and degradation diagnosis in polyethylene and polyimide", IEEE Conf. Properties Appl. Dielectr. Materials, Brisbane, Australia, pp. 630-633, 1994

[21] H. Okubo, N. Hayakawa, and A. Matsushita, "The relationship between partial discharge current pulse waveforms and physical mechanisms", IEEE Electr. Insul. Mag., Vol. 18, No.3, pp. 38-45, 2002.

[22] H. Okubo, N. Hayakawa, "A novel technique for partial discharge and breakdown investigation based on current pulse waveform analysis", IEEE Trans. Dielectr. Electr. Insul., Vol. 12, No.4, pp. 736-744, 2005.

[23] H. Okubo, "Enhancement of electrical insulation performance in power equipment based on dielectric material properties", IEEE Trans. Dielectr. Electr. Insul., Vol.19, No.3, pp. 733-754, 2012. 
[24] M. Florkowski and B. Florkowska, "Phase-resolved rise-time-based discrimination of partial discharges", IET Gen. Transm. Distrib., Vol. 3, No.1, pp. 115-124, 2009.

[25] C. Hudon and B. Mario, "Partial discharge signal interpretation for generator diagnostics", IEEE Trans. Dielectr. Electr. Insul., Vol. 12, No.2, pp. 297-319, 2005.

[26] A. Cavallini, A. Contin, G. C. Montanari, and F. Puletti, "Application of a new methodology for identification of PD in electrical apparatus", IEEE Trans. Dielectr. Electr. Insul., Vol. 12, No.2, pp. 203-215, 2005.

[27] J. Li, C. Sun, and S. Grzybowski. "Partial discharge image recognition influenced by fractal image compression", IEEE Trans. Dielectr. Electr. Insul., Vol. 15, No.2, pp. 496-504, 2008.

[28] R. Liao, J. Yan, L. Yang, M. Zhu, and B. Liu, "Study on the relationship between damage of oil-impregnated insulation paper and evolution of phase-resolved partial discharge patterns", Europ. Trans. Electr. Power, Vol. 21, No.3, pp. 2112-2124, 2011.

[29] H. Illias, G. Chen, and P. L. Lewin. "Partial discharge behavior within a spherical cavity in a solid dielectric material as a function of frequency and amplitude of the applied voltage", IEEE Trans. Dielectr. Electr. Insul., Vol. 18, No.2, pp. 432-443, 2011.

[30] H. G. Kranz, "Diagnosis of partial discharge signals using neural networks and minimum distance classification", IEEE Trans. Electr. Insul., Vol. 28, No.6, pp. 1016-1024, 1993.

[31] L, Hao, P. L. Lewin, J. A. Hunter, D. J. Swaffield, A. Contin, C. Walton, and M. Michel, "Discrimination of multiple PD sources using wavelet decomposition and principal component analysis", IEEE Trans. Dielectr. Electr. Insul., Vol. 18, No.5, pp. 1702-1711, 2011.

[32] J. Li, S. Grzybowski, Y. Sun, and X. Chen, "Dielectric properties of rapeseed oil paper insulation", IEEE Conf. Electr. Insul. Dielectr. Phenomena (CEIDP), Vancouver, British Columbia, Canada, pp. 500503, 2007.

[33] ASTM Standard D149-09: "Test Method for Dielectric Breakdown Voltage and Dielectric Strength of Solid Electrical Insulating Materials at Commercial Power Frequencies", 2009.

[34] W. Chen, X. Chen, S. Peng, J. Li, "Canonical correlation between partial discharges and gas formation in transformer oil paper insulation", Energies, Vol. 5, No. 4, pp. 1081-1097, 2012.

[35] IEC 61294: "Insulating liquids-determination of the partial discharge inception voltage (PDIV) - test procedure", 1993.

[36] C. S. Kim, T. Kondo, and T. Mizutani, "Change in PD pattern with aging”, IEEE Trans. Dielectr. Electr. Insul., Vol. 11, No.1, pp. 13-18, 2004.

[37] R. Bartnikas, "A comment concerning the rise time of partial discharge pulses”, IEEE Trans. Dielectr. Electr. Insul., Vol. 12, No.2, pp. 196-202, 2005.

[38] M. Pompili and R. Bartnikas, "On partial discharge measurement in dielectric liquid”, IEEE Trans. Dielectr. Electr. Insul., Vol. 19, No.5, pp 1476-1481, 2012.

[39] K. X. Lai, B. T. Phung, T. R. Blackburn, and N. A. Muhamad, "Comparison of partial discharge activity in mineral oil and biodegradable oil", IEEE Conf. AUPEC, pp. 1-6, 2007.

[40] J. Yan, R. Liao, L. Yang, and J. Li, "Study on microstructure and electrical properties of oil-impregnated paper insulation after exposure to partial discharge", Europ. Trans. Electr. Power, Vol. 22, No.6, pp. $733-$ 746, 2012.

[41] L. Niemeyer, "A generalized approach to partial discharge modeling", IEEE Trans. Dielectr. Electr. Insul., Vol. 2, No.4, pp. 510-528, 1995.

[42] Y. Wei, M. Zhu, Y. Li, L. Zhao, J. Deng, H. Mu, and G. Zhang, "Partial discharge characteristics and trap parameters analysis of aged oilimpregnated paper", IEEE Trans. Dielectr. Electr. Insul., Vol. 22, No.6, pp. 3442-3450, 2015.

[43] R. Liao, J. Hao, G. Chen, Z. Ma, and L. Yang, "A comparative study of physicochemical, dielectric and thermal properties of pressboard insulation impregnated with natural ester and mineral oil", IEEE Trans. Dielectr. Electr. Insul., Vol. 18, No.5, pp. 1626-1637, 2011.

[44] I. H. Suwarno, Y. Suzuoki, T. Mizutani, and K. Uchida, "Partial discharge patterns of electrical treeing in polyethylene", IEEE Conf. Properties Appl. Dielectr. Materials, Brisbane, Australia, pp. 379-382, 1994.

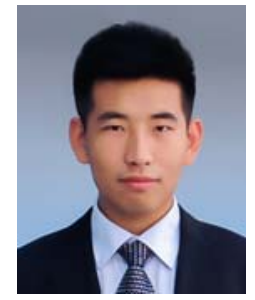

Lu Cui was born in Shandong, China in August 1993. He received the B.Sc. degree from Chongqing University, Chongqing, China in 2013 and is currently pursuing the Ph.D. degree in the Department of Electrical Engineering at Chongqing University. From 2015 to 2016, as a Ph.D. student, he works on the thermal and electrical properties of vegetable oil/pressboard insulation system in University of Southampton, UK. His main research interests include partial discharge characteristic analysis, dissolved gas analysis and diagnosis of vegetable oil/pressboard insulation for power transformer.

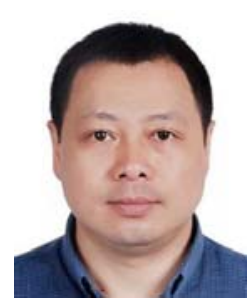

Weigen Chen was born in Zhejiang, China in August 1967. He received his B.Sc., M.Sc. and Ph.D. degree in Electrical Engineering from Chongqing University, China in 1990, 1993, and 2003 respectively. Currently he is a Professor in the Department of Electrical Engineering at Chongqing University. His main research interests include online monitoring and fault diagnosis of power equipment, condition-based maintenance, and the internal insulation and thermal properties of power transformers. He is a member of CIGRÉ WG A2.27 working groups. Dr. Chen has published two books and over 130 papers in his professional work.

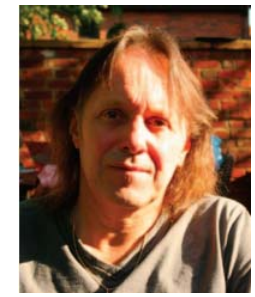

Alun Vaughan (M'06-SM'07) has a B.Sc. degree in chemical physics and a Ph.D. degree in polymer physics. After working at the UK's Central Electricity Research Laboratories and spending a period as an academic at The University of Reading, he is now Professor of Dielectric Materials and Head of the Electronic and Electrical Engineering research group at the University of Southampton. He is a former chair of The Dielectrics Group of the Institute of Physics, a Fellow of the Institute of Physics, and a

Fellow of the IET.

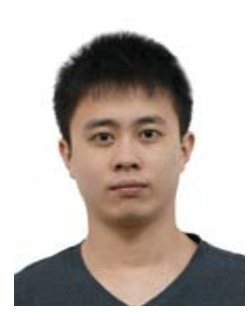

Bo Xie was born in Sichuan, China in September 1989. He received the B.Sc. degree from Chongqing University, Chongqing, China in 2011 and is currently pursuing the Ph.D. degree in the Department of Electrical Engineering at Chongqing University. His main research interests include partial discharge on-line monitoring, and fault diagnosis technology of transformer.

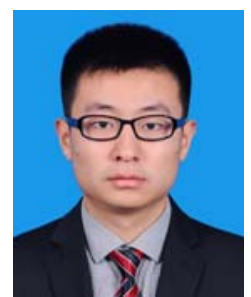

Zhenze Long was born in Chongqing, China in November 1989. He received dual M.Sc. Degree from Department of Electrical Engineering, Chongqing University, Chongqing, China and University of Wisconsin Milwaukee, USA in 2015. Now he works for Sichuan Electric Power Research Institute, State Grid, China. His main research interests include partial discharge diagnosis, high voltage test and big data analysis 